\section{Autism Spectrum Disorder in the COVID 19 Era: New Challenges - New Solutions}

This survey explored the support available and the effect of lockdown on children with autism spectrum disorder and their families in India and the United Kingdom. Our findings showed significant problems for children and families due to lockdown. App-based information delivered to parents with support showed encouraging feedback.

Keywords: Anxiety behavior, App-based support, Lockdown, Telehealth.

Published online: June 28, 2021; PII: S097475591600351

During the coronavirus disease 2019 (COVID-19) lockdown, all face-to-face support and planned activities of children with ASD were interrupted. This brought a significant change of routine for them [1]. They reported higher levels of anxiety, reduced dispositional hope, and psychological well-being [2]. Information technologies have been an essential tool during these challenging times [3]. Studies have reported positive effects of parental empowerment with training and support using technological platforms [4]. We investigate the impact of lockdown on children with ASD and their families in India and the UK.

Parents of 45 children with a confirmed diagnosis of ASD 15 from Conwall, United Kingdom [mean (range) age, 11.06 (7.02-17.05) years] and 30 from Lucknow, India [mean (range) age, 5.2 (2.11- 8.07) years] participated in the survey. Children with confirmed ASD were identified from clinical records of the child development center in Lucknow and Cornwall. Consent was obtained and General Data Protection Regulation guidelines were followed during data collection and analysis.

A semi-structured survey was constructed. It included four variables: change in behavior (CIB), change in the routine (CIR), regression in skills (RIS), and parental stress (PS) (Web Fig. 1). The final survey was reviewed by an expert panel, and their suggestions were included before conducting the telephonic survey. Speech and language therapists in the UK and trained child psychologists in India conducted telephonic surveys in mid of April over two weeks. Each interview took approximately 45 minutes. All questions on CIB, CIR and RIS were rated on an 8-point scale ranging from 0 to 7 and for PS on a 5-point scale ranging from 0 to 4 .

Nonparametric $t$-test, Pearson correlation and multivariate regression analysis were calculated to measure the difference, bivariate associations, and the impact of lockdown on the study variables.

The mean rank for CIR, PS and CIS were higher in the UK as compared to India, whereas there was no significant difference in CIB. CIR and CIB were reported for all children. All parents reported increased PS (Table I). There was a significant correlation of change in behavior score with change in routine score $(\mathrm{r}=0.446, P<0.01)$, regression in skills score $(r=0.750, P<0.01)$ and parental stress score $(r=0.370, P<0.05)$. Parental stress score also significantly correlated with change in routine $(r=0.535, P<0.01)$ and with regression in skills $(r=0.375, P<0.05)$. Regression in skill and change in routine scores were also significantly correlated $(r=0.410, P<0.01)$.

Parents and families reported extra difficulties in managing adults with ASD $[5,6]$. In the UK, children with ASD are provided diagnosis based on NICE guidelines. Necessary support is provided through schools, along with parental courses offered by the local council. Additional help is available through pediatricians, speech and language, child and adolescent mental health services, occupational therapy, and support groups. In Lucknow, children were assessed by a multiprofessional team. The diagnosis was confirmed using ADI-R. The families who enroll in the early intervention program were provided with a home program support app.

Disruption in routine due to lockdown affected the behavior of children with ASD [1]. Change in routine was positively and significantly correlated with change in behavior, regression in skills, and parental stress, similar to previous reports [5].

In Cornwall, the parents' interview explained a lack of support or contact because of the long waiting list of appointments with professionals before the pandemic, which worsened with the lockdown. Studies reported a lack of support for both autistic individuals and their family's post-diagnosis prior to lockdown $[8,9]$.

The mainstay of autism treatment is parental training and environmental modification around the child [10]. This study identified a gap in empowering parents with resources postdiagnosis that they can access when required. The app model support has shown promise, but parents needed support,

Table I Impact of Lockdown on Children With Autism Spectrum Disorder and Their Families in India and United Kingdom

\begin{tabular}{llr}
\hline Group & Mean rank(SD) & P value \\
\hline Change in behavior & & \\
India & $24.0(10.49)$ & 0.45 \\
UK & $20.9(10.49)$ & \\
Change in the routine & & \\
India & $18.22(4.44)$ & \\
UK & $32.57(4.44)$ & \\
Regression in skills & & \\
India & $22.28(2.84)$ & \\
UK & & \\
Parental stress & $24.43(2.84)$ & \\
India & & \\
UK & $17.18(6.30)$ & \\
\hline
\end{tabular}

Number of families enrolled UK-15, India-30. Proportion of parents who reported changes in the variable during lockdown was 100\% for all variables except ${ }^{a} 78.6 \%$ for regression in skills from UK. 
despite using the app. Due to the lack of appropriate measures, we were unable to measure the psychometric of the survey. However, the finding of this study is based on the direct parent's experiences, which is a strength of the study. The result helped in re-establishing the important role of routine and structure in children with ASD (Web Table I).

In conclusion, COVID-19 lockdown brought an opportunity to reassess our services for children with ASD. Technology-mediated support showed encouraging feedback of being used in combination with clinical practice.

Acknowledgements: Rohan Pandey and Shipra Verma for helping in conducting the survey in India.

Note: Additional material related to this study is available with the online version at $w w w$.indianpediatrics.net

Ethics approval: Royal Cornwall Hospitals NHS trust Audit department, ID 1721.

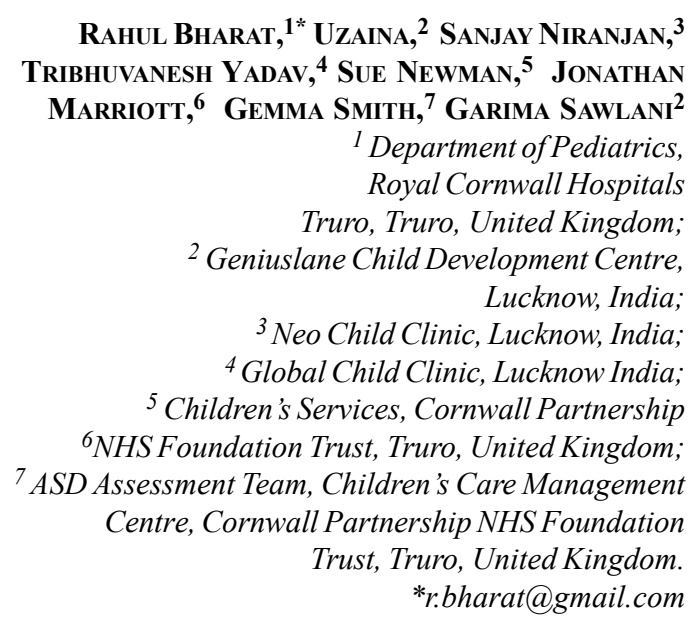

RAhUl Bharat, ${ }^{1 *}$ Uzaina, ${ }^{2}$ Sanjay Niranjan, ${ }^{3}$ Tribhuvanesh Yadav, ${ }^{4}$ Sue Newman, ${ }^{5}$ Jonathan Marriott, ${ }^{6}$ Gemma SMith, ${ }^{7}$ Garima SaWlani ${ }^{2}$

${ }^{1}$ Department of Pediatrics, Royal Cornwall Hospitals Truro, Truro, United Kingdom; ${ }^{2}$ Geniuslane Child Development Centre, Lucknow, India;

${ }^{3}$ Neo Child Clinic, Lucknow, India; ${ }^{4}$ Global Child Clinic, Lucknow India;

${ }^{5}$ Children's Services, Cornwall Partnership ${ }^{6}$ NHS Foundation Trust, Truro, United Kingdom; ${ }^{7}$ ASD Assessment Team, Children's Care Management Centre, Cornwall Partnership NHS Foundation Trust, Truro, United Kingdom. *r.bharat@gmail.com

\section{REFERENCES}

1. Colizzi M, Sironi E, Antonini F, et al. Psychosocial and behavioral impact of COVID-19 in autism spectrum disorder: An online parent survey. Brain Sci. 2020;10:341.

2. Ersoy K, Altýn B, Bayram SB, Güngörmüp OO. The comparison of impact of health anxiety on dispositional hope and psychological well-being of mothers who have children diagnosed with autism and mothers who have normal children in Covid-19 pandemic [Turkish]. Sosyal Bilimler Araptýrma Dergisi. 2020;9:117-26.

3. Eshraghi AA, Li C, Alessandri M, et al. COVID19: overcoming the challenges faced by individuals with autism and their families. Lancet Psychiat. 2020;7:481-83.

4. Lai MC, Anagnostou E, Wiznitzer M, et al. Evidence-based support for autistic people across the lifespan: Maximizing potential, minimizing barriers, and optimizing the personenvironment fit. Lancet Neurol. 2020;19:434-51.

5. White LC, Law JK, Daniels AM, et al. Impact of COVID-19 on individuals with ASD and their caregivers: A perspective from the SPARK cohort. J Autism Dev Disord. 2021:1-8 [Epub ahead of print]

6. Singh S, Roy D, Sinha K, et al. Impact of COVID-19 and lockdown on mental health of children and adolescents: A narrative review with recommendations. Psychiat Res. 2020;293: 113429.

7. Davis NO, Carter AS. Parenting stress in mothers and fathers of toddlers with autism spectrum disorders: Associations with child characteristics. J Autism Dev Disord. 2008;38:1278.

8. Crane L, Batty R, Adeyinka H, et al. Autism diagnosis in the United Kingdom: Perspectives of autistic adults, parents and professionals. J Autism Dev Disord. 2018;48:3761-72.

9. Jones L, Goddard L, Hill EL, et al. Experiences of receiving a diagnosis of autism spectrum disorder: a survey of adults in the United Kingdom. J Autism Dev Disord. 2014;44:3033-44.

10. Beaudoin AJ, Sébire G, Couture M. Parent training interventions for toddlers with autism spectrum disorder. Autism Res Treat. 2014;2014:839890.

\section{CLIPPINGS}

$\theta$

Treatment of infantile-onset spinal muscular atrophy with nusinersen: final report of a phase 2, open-label, multicentre, dose-escalation study (Lancet Child Adolesc Health. 2021;5:491-500)

Spinal muscular atrophy (SMA) is a fairly common autosomal recessive, neurodegenerative disease caused by biallelic mutations in the survival motor neuron 1 (SMN1) gene. SMA is characterized by motor neuron degeneration, resulting in progressive muscle weakness, immobility and appreciable morbidities and mortality. Currently, three disease modifying therapeutic options are approved for treatment: Nusinersen (Spinraza), the antisense oligonucleotide given through intrathecal route; Risdiplam, an orally administered splicing modifier of motor neuron 2 (SMN2) and Onasemnogeneabeparvovec (Zolgensma), an adeno-associated virus-based gene replacement therapy.

Nusinersen is the oldest and the most well studied medication among them. It had showed a favourable benefit-risk profile in participants with infantile-onset spinal muscular atrophy at the interim analysis of a phase 2 clinical study. In the above study, authors present the final analysis, assessing the efficacy and safety of nusinersen over 3 years. It recruited 20 symptomatic infants aged between 3 weeks and 6 months with two or three SMN2 gene copies, between May 3, 2013, and July 9,2014 . Participants received multiple intrathecal loading doses of $6 \mathrm{mg}$ equivalent nusinersen (cohort 1) or $12 \mathrm{mg}$ dose equivalent (cohort 2), followed by maintenance doses of $12 \mathrm{mg}$ equivalent nusinersen. Median time on study was $36 \cdot 2$ months. In the 13 participants with two SMN2 copies treated with 12 $\mathrm{mg}$ nusinersen, the HINE-2 motor milestone total score increased steadily from a baseline mean (SD) of $1.46(0.52)$ to $11.86(6 \cdot 18)$ at day 1135 , representing significant clinical improvement. At study closure (Aug 21, 2017), 15 (75\%) of 20 participants were alive. All five deaths (one in cohort 1 and four in cohort 2) were likely to be related to spinal muscular atrophy disease progression.

KAUSIK MANDAL kausik@sgpgi.ac.in 